\title{
New Fastest Linearly Independent Transforms over GF(3)
}

\author{
Bogdan J. Falkowski and Cicilia C. Lozano \\ School of Electrical and Electronic Engineering \\ Nanyang Technological University \\ Block S1, 50 Nanyang Avenue, Singapore 639798
}

\author{
Tadeusz Łuba \\ Institute of Telecommunications \\ Warsaw University of Technology \\ Nowowiejska 15/19, 00-665 Warsaw, Poland
}

\begin{abstract}
New fastest linearly independent (LI) transforms for ternary functions are introduced in this paper. The transforms operate over Galois Field (3) (GF(3)) and have smaller computational costs than ternary ReedMuller transform. The new transforms are built based on the known fastest LI transforms over GF(3) and the relations between them are shown. Several properties for the new transforms are presented. Experimental results for the new transforms are also listed and compared with the known fastest LI transforms over GF(3).
\end{abstract}

\section{Introduction}

Ternary switching functions are mappings $f:\{0,1,2\}^{n} \rightarrow\{0,1,2\}$ and can be considered in different algebraic structures with various polynomial expansions or spectral transforms. To develop the spectral transform theory for switching ternary functions, one needs to refer to abstract harmonic analysis that is a mathematical approach derived from the classical Fourier analysis [1]. Developments in harmonic analysis on finite Abelian groups resulted in various transforms such as Walsh, Reed-Muller, and their possible modifications that have many attractive features and are useful in applications such as a new theory of nonlinear signal and image processing [2-4] and many other areas of applied mathematics [5].

Fastest linearly independent (LI) transforms over Galois Field (3) (GF(3)) have the simplest butterfly diagrams of all possible LI transforms over GF(3). In this paper, new fastest LI transforms over $\mathrm{GF}(3)$ and their properties are investigated and compared with the known fastest LI transforms over GF(3) [6]. The presented transforms and their properties shown here can be used as bases for analysis, synthesis, and testing of ternary functions as well as creating their spectral decision diagrams in a similar manner as for other polynomial expansions $[1,7,8]$.

\section{Basic definitions}

Definition 1. Let $M_{n}$ be an $N \times N\left(N=3^{n}\right)$ matrix with rows corresponding to minterms and columns corresponding to some ternary switching functions of $n$ variables. If the sets of columns are linearly independent over GF(3), then $M_{n}$ has only one inverse in GF(3) and is said to be linearly independent.

Definition 2. Let $M_{n}$ be an LI matrix of order $N=3^{n}$ as specified in Definition 1 and $\vec{F}=\left[F_{0}, F_{1}, \ldots, F_{N-1}\right]^{T}$ be the truth column vector of an $n$-variable ternary switching function $f\left(\overrightarrow{x_{n}}\right)$ in a natural ternary ordering. Then,

and

$$
\begin{aligned}
& \vec{A}=M_{n}^{-1} \vec{F} \\
& \vec{F}=M_{n} \vec{A},
\end{aligned}
$$

where $\vec{A}=\left[A_{0}, A_{1}, \ldots, A_{N-1}\right]^{T}$ represents the spectrum of $f\left(\overrightarrow{x_{n}}\right)$ based on $M_{n}, M_{n}^{-1}$ is the inverse of $M_{n}$ over GF(3), $T$ denotes transpose operator, and all the additions and multiplications are performed over $\mathrm{GF}(3)$.

Definition 3. Let $f\left(\overrightarrow{x_{n}}\right)$ be an $n$-variable ternary switching function. Then by Definitions 1 and 2 the LI expansion of $f\left(\overrightarrow{x_{n}}\right)$ based on a ternary LI transform $M_{n}$ can be written as

$$
f\left(\overrightarrow{x_{n}}\right)=\sum_{j=0}^{3^{n}-1} A_{j} g_{j},
$$

where $g_{j}\left(0 \leq j \leq 3^{n}-1\right)$ denotes the ternary switching function whose truth vector is given by column $j$ of $M_{n}$ and $A_{j}$ denotes the $j$-th spectral coefficient in the spectrum of $f\left(\overrightarrow{x_{n}}\right)$ based on $M_{n}$. The additions and multiplications inside (3) are evaluated over GF(3).

Groups of ternary LI transforms with lower computational cost than ternary Reed-Muller transform have been presented in [6] where they are classified into classes Y1, Y2, Z1, and Z2 based on their structure. There 
are six ternary LI transforms inside each class, where four of them have the same computational costs that are lower than the computational cost of the other two transforms in the class. In this paper, only those ternary fastest LI transforms with the lowest computational cost are discussed. Together, we refer to those transforms as known ternary fastest LI transforms.

All the known ternary fastest LI transforms presented in [6] are recursive and can be defined in terms of the submatrices $O_{n-1}, I_{n-1}, J_{n-1}, Y_{n-1}$ or $M_{n-1}$, where $O_{n-1}$ denotes a $3^{n-1} \times 3^{n-1}$ submatrix with all its elements being zero, $I_{n-1}$ denotes the identity submatrix of size $3^{n-1} \times 3^{n-1}, \quad J_{n-1}$ denotes the reverse identity matrix of dimension $3^{n-1} \times 3^{n-1}$, and $Y_{n-1}$ denotes a $3^{n-1} \times 3^{n-1}$ submatrix with all its elements being zero except for one element located at one corner of each matrix, depending on the location of $Y_{n-1}$. Their recursive definitions have the following general form:

$$
M_{n}=\left[\begin{array}{ccc}
M_{n-1}^{(1)} & O_{n-1} & M_{n-1}^{(2)} \\
O_{n-1} & M_{n-1}^{(5)} & O_{n-1} \\
M_{n-1}^{(3)} & O_{n-1} & M_{n-1}^{(4)}
\end{array}\right],
$$

where each submatrix $M_{n-1}^{(j)}, j=\{1,2,3,4,5\}$, has a dimension of $3^{n-1} \times 3^{n-1}$ and contains one recursive equation which is either $O_{n-1}, X_{n-1}, Y_{n-1}$ or $M_{n-1}$, where $X_{n-1}=I_{n-1}$ or $J_{n-1}$.

Definition 4. There are four ternary fastest LI transforms with the lowest computational cost in class Y1 [6]. Their forward transforms are defined recursively as

$$
\begin{aligned}
& M_{(Y 1.1) n}= {\left[\begin{array}{lll}
M_{n-1} & O_{n-1} & O_{n-1} \\
O_{n-1} & I_{n-1} & O_{n-1} \\
Y_{n-1} & O_{n-1} & M_{n-1}
\end{array}\right] } \\
& M_{(Y 1.2) n}= {\left[\begin{array}{lll}
M_{n-1} & O_{n-1} & O_{n-1} \\
O_{n-1} & J_{n-1} & O_{n-1} \\
Y_{n-1} & O_{n-1} & M_{n-1}
\end{array}\right] } \\
& M_{(Y 1.4) n}=\left[\begin{array}{lll}
M_{n-1} & O_{n-1} & Y_{n-1} \\
O_{n-1} & I_{n-1} & O_{n-1} \\
O_{n-1} & O_{n-1} & M_{n-1}
\end{array}\right] \\
& M_{(Y 1.5) n}=\left[\begin{array}{lll}
M_{n-1} & O_{n-1} & Y_{n-1} \\
O_{n-1} & J_{n-1} & O_{n-1} \\
O_{n-1} & O_{n-1} & M_{n-1}
\end{array}\right],
\end{aligned}
$$

where $O_{n-1}, I_{n-1}, J_{n-1}, Y_{n-1}$, and $M_{n-1}$ have been defined before. Their inverse transforms can be obtained by simply replacing $Y_{n-1}$ and $M_{n-1}$ in the forward transform with $2 Y_{n-1}$ and $M_{n-1}^{-1}$, respectively as shown in (9)-(12).

$$
\begin{aligned}
M_{(Y 1.1) n}^{-1}= & {\left[\begin{array}{lll}
M_{n-1}^{-1} & O_{n-1} & O_{n-1} \\
O_{n-1} & I_{n-1} & O_{n-1} \\
2 Y_{n-1} & O_{n-1} & M_{n-1}^{-1}
\end{array}\right] } \\
M_{(Y 1.2) n}^{-1}= & {\left[\begin{array}{lll}
M_{n-1}^{-1} & O_{n-1} & O_{n-1} \\
O_{n-1} & J_{n-1} & O_{n-1} \\
2 Y_{n-1} & O_{n-1} & M_{n-1}^{-1}
\end{array}\right] } \\
M_{(Y 1.4) n}^{-1}= & {\left[\begin{array}{lll}
M_{n-1}^{-1} & O_{n-1} & 2 Y_{n-1} \\
O_{n-1} & I_{n-1} & O_{n-1} \\
O_{n-1} & O_{n-1} & M_{n-1}^{-1}
\end{array}\right] } \\
M_{(Y 1.5) n}^{-1}= & {\left[\begin{array}{lll}
M_{n-1}^{-1} & O_{n-1} & 2 Y_{n-1} \\
O_{n-1} & J_{n-1} & O_{n-1} \\
O_{n-1} & O_{n-1} & M_{n-1}^{-1}
\end{array}\right] . }
\end{aligned}
$$

Definition 5. The operator $R_{1, r}$ on a $3^{n} \times 3^{n}$ matrix $M_{n}$ is defined as performing $9^{n-r-1}$ counterclockwise rotations twice involving $\left(8 \cdot 9^{n-r-1}\right)$ submatrices each of order $3^{r}(0 \leq r \leq n-1)$.

Example 1. Let $M_{2}=\left[\begin{array}{lllllllll}1 & 0 & 1 & 0 & 0 & 0 & 0 & 0 & 0 \\ 0 & 1 & 0 & 0 & 0 & 0 & 0 & 0 & 0 \\ 1 & 0 & 0 & 0 & 0 & 0 & 0 & 0 & 0 \\ 0 & 0 & 0 & 0 & 0 & 1 & 0 & 0 & 0 \\ 0 & 0 & 0 & 0 & 1 & 0 & 0 & 0 & 0 \\ 0 & 0 & 0 & 1 & 0 & 0 & 0 & 0 & 0 \\ 1 & 0 & 0 & 0 & 0 & 0 & 1 & 0 & 1 \\ 0 & 0 & 0 & 0 & 0 & 0 & 0 & 1 & 0 \\ 0 & 0 & 0 & 0 & 0 & 0 & 1 & 0 & 0\end{array}\right]$. Then,

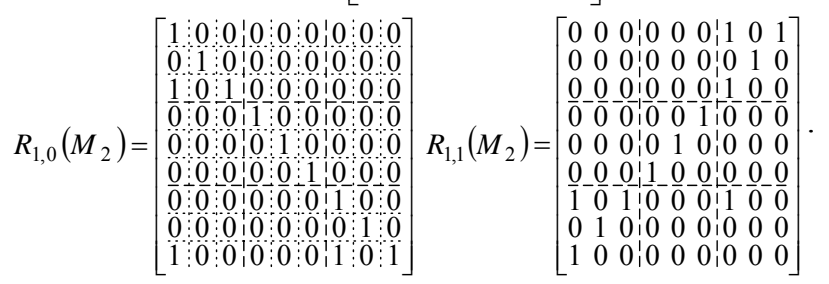

Definition 6. The operator $R_{2}$ on a $3^{n} \times 3^{n}$ matrix $M_{n}$ is defined as recursively applying operator $R_{1, r}$ on $M_{n}$ for $r$ $=n-1, n-2, \ldots, 0$. The square of operator $R_{2}$ is specified as $R_{2}^{2}\left(M_{n}\right)=R_{2}\left(R_{2}\left(M_{n}\right)\right)$.

Definition 7. The forward and inverse ternary fastest LI transforms $M_{(Y 1 . \theta) n}$ and $\left(M_{(Y 1 . \theta) n}\right)^{-1}$ can be calculated by fast transform by representing them in the following factorized form

$$
M_{(Y 1 . \theta) n}=\prod_{j=n}^{1} K_{n, j}^{\theta}
$$


and

$$
\left(M_{(Y 1 . \theta) n}\right)^{-1}=\prod_{j=n}^{1}\left(K_{n, j}^{\theta}\right)^{-1}
$$

where $K_{n, j}^{\theta}$ and $\left(K_{n, j}^{\theta}\right)^{-1}$ denote the $j$-th factorized transform matrix of $M_{(Y 1 . \theta) n}$ and $\left(M_{(Y 1 . \theta) n}\right)^{-1}$, respectively $(1 \leq j \leq n, \theta \in\{1,2,4,5\})$. Property 1 gives the general formulae for $K_{n, j}^{\theta}$ and $\left(K_{n, j}^{\theta}\right)^{-1}$.

Property 1. The factorized transform matrices of $M_{(Y 1 . \theta) n}$ and $\left(M_{(Y 1 . \theta) n}\right)^{-1}(\theta \in\{1,2,4,5\})$ can be derived by using (15)-(22) as follows:

$$
\begin{aligned}
& K_{n, j}^{1}=\left[\begin{array}{ccc}
K_{n-1, j}^{1} & O_{n-1} & O_{n-1} \\
O_{n-1} & I_{n-1} & O_{n-1} \\
O_{n-1} & O_{n-1} & K_{n-1, j}^{1}
\end{array}\right] \\
& \left(K_{n, j}^{1}\right)^{-1}=\left[\begin{array}{ccc}
\left(K_{n-1, j}^{1}\right)^{-1} & O_{n-1} & O_{n-1} \\
O_{n-1} & I_{n-1} & O_{n-1} \\
O_{n-1} & O_{n-1} & \left(K_{n-1, j}^{1}\right)^{-1}
\end{array}\right] \\
& K_{n, j}^{2}=\left[\begin{array}{ccc}
K_{n-1, j}^{2} & O_{n-1} & O_{n-1} \\
O_{n-1} & X_{n-1} & O_{n-1} \\
O_{n-1} & O_{n-1} & K_{n-1, j}^{2}
\end{array}\right] \\
& \left(K_{n, j}^{2}\right)^{-1}=\left[\begin{array}{ccc}
\left(K_{n-1, j}^{2}\right)^{-1} & O_{n-1} & O_{n-1} \\
O_{n-1} & X_{n-1} & O_{n-1} \\
O_{n-1} & O_{n-1} & \left(K_{n-1, j}^{2}\right)^{-1}
\end{array}\right] \\
& K_{n, j}^{4}=R_{2}^{2}\left(K_{n, j}^{1}\right) \\
& \left(K_{n, j}^{4}\right)^{-1}=R_{2}{ }^{2}\left(\left(K_{n, j}^{1}\right)^{-1}\right) \\
& K_{n, j}^{5}=R_{2}^{2}\left(K_{n, j}^{2}\right) \\
& \left(K_{n, j}^{5}\right)^{-1}=R_{2}^{2}\left(\left(K_{n, j}^{2}\right)^{-1}\right),
\end{aligned}
$$

where $K_{j, j}^{\theta}$ represents the identity matrix of size $3^{j} \times 3^{j}$ with bottom left element replaced by ' 1 ', $\left(K_{j, j}^{\theta}\right)^{-1}$ represents the identity matrix of size $3^{j} \times 3^{j}$ with bottom left element replaced by ' 2 ' $(\theta \in\{1,2\})$, and $X_{n-1}=\left\{\begin{array}{ll}J_{n-1}, & \text { if } n=j+1 \\ I_{n-1}, & \text { otherwise. }\end{array}\right.$ Note that $O_{n-1}, I_{n-1}$, and $J_{n-1}$ in (15)-(18) are the same as in (5)-(12).

Fig. 1 shows the forward and inverse butterfly diagrams for fast computation of the ternary fastest LI transforms $M_{(Y 1.1) 2}$ based on Definition 7 and Property 1. The same butterfly diagrams for the LI transform $M_{(Y 1.2) 2}$ are shown in Fig. 2. In both figures, the solid and dashed line represents the values 1 and 2 , respectively.

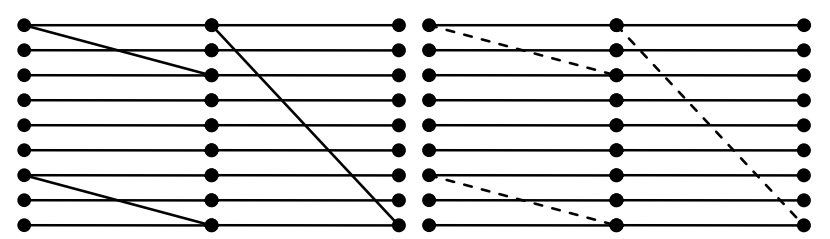

(a)

(b)

Figure 1. Butterfly diagrams of $M_{(Y 1.1) 2}$ : (a) Forward transform; (b) Inverse transform.

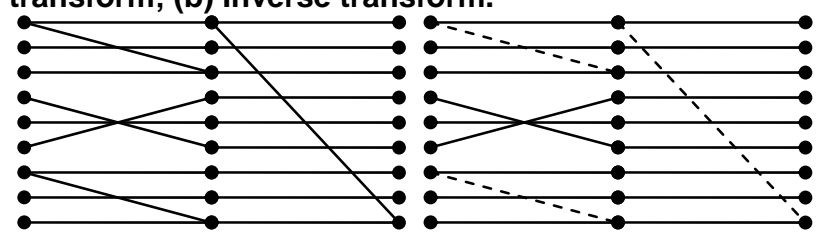

(a)

(b)

Figure 2. Butterfly diagrams of $M_{(Y 1.2) 2}$ : (a) Forward transform; (b) Inverse transform.

Property 2[6]. The number of additions required to compute the spectra of ternary fastest LI transforms $M_{(Y 1 . \theta) n}(\theta \in\{1,2,4,5\})$ is $2^{n}-1$.

\section{Ternary fastest LI transforms with permutation}

In this paper, we want to identify new ternary fastest LI transforms that have the same computational cost as the known ternary fastest LI transforms and can also be calculated efficiently by fast transforms while offering the possibility of more compact polynomial representations, i.e., have the smaller number of nonzero terms. One of the simplest ways to do that is by permuting the known ternary fastest LI transforms. Such class of ternary fastest LI transforms is defined in this Section. It should be noted that due to the relations between the known ternary fastest LI transforms, the LI expansions based on all the ternary fastest LI transforms with permutation cover the LI expansions based on all the known ternary fastest LI transforms. As such, the minimum number of nonzero spectral coefficients in the spectra of ternary fastest LI transforms with permutation is always smaller than or equal to the minimum nonzero spectral coefficient number in the spectra of all the known ternary fastest LI transforms. 
Permutation matrices are matrices that contain exactly one ' 1 ' in each row and column. As such there are six possible permutation matrices of size $3 \times 3$ and $6^{n}$ permutation matrices of size $3^{n} \times 3^{n}$ that can be derived from the Kronecker product of the $3 \times 3$ permutation matrices.

Definition 8. Let the six $3 \times 3$ permutation matrices be denoted by $\rho_{0}, \rho_{1}, \rho_{2}, \rho_{3}, \rho_{4}$, and $\rho_{5}$, where $\rho_{0}=\left[\begin{array}{lll}1 & 0 & 0 \\ 0 & 1 & 0 \\ 0 & 0 & 1\end{array}\right], \quad \rho_{1}=\left[\begin{array}{lll}1 & 0 & 0 \\ 0 & 0 & 1 \\ 0 & 1 & 0\end{array}\right], \quad \rho_{2}=\left[\begin{array}{lll}0 & 1 & 0 \\ 1 & 0 & 0 \\ 0 & 0 & 1\end{array}\right]$, $\rho_{3}=\left[\begin{array}{lll}0 & 1 & 0 \\ 0 & 0 & 1 \\ 1 & 0 & 0\end{array}\right], \rho_{4}=\left[\begin{array}{lll}0 & 0 & 1 \\ 1 & 0 & 0 \\ 0 & 1 & 0\end{array}\right]$, and $\rho_{5}=\left[\begin{array}{lll}0 & 0 & 1 \\ 0 & 1 & 0 \\ 1 & 0 & 0\end{array}\right]$. Then $P_{n}^{p}$ is defined as the permutation matrix of size $3^{n} \times 3^{n}$ with permutation number $p \quad\left(0 \leq p \leq 6^{n}-1\right)$ that is calculated by

$$
P_{n}^{p}=\bigotimes_{j=n}^{1} \rho_{p_{j}},
$$

where $<p>_{6}=<p_{n}, p_{n-1}, \ldots, p_{1}>$ is the $n$-digit sixvalued representation of $p$ and $\otimes$ denotes Kronecker product $[1,2,4,5,7]$.

Due to the property of Kronecker product, the inverse of $P_{n}^{p}$, denoted by $\left(P_{n}^{p}\right)^{-1}$, is simply

$$
\left(P_{n}^{p}\right)^{-1}=\underset{j=n}{\otimes}\left(\rho_{p_{j}}\right)^{-1}
$$

where $\rho_{3}$ and $\rho_{4}$ are inverses of each other and $\rho_{0}, \rho_{1}$, $\rho_{2}$, and $\rho_{5}$ are self inverse.

Definition 9. Let $M_{n}(p)$ denote ternary fastest LI transform matrix of size $3^{n} \times 3^{n}$ with permutation number $p \quad\left(0 \leq p \leq 6^{n}-1\right)$. Then $M_{n}(p)$ and its inverse transform matrix $\left(M_{n}(p)\right)^{-1}$ are defined as

$$
M_{n}(p)=P_{n}^{p} \cdot \prod_{j=n}^{1} K_{n, j}^{1}
$$

and

$$
\left(M_{n}(p)\right)^{-1}=\left(\prod_{j=n}^{1}\left(K_{n, j}^{1}\right)^{-1}\right) \cdot\left(P_{n}^{p}\right)^{-1}
$$

respectively.

Property 3. There are altogether $3^{n}+2^{n}-1$ nonzero elements inside both $M_{n}(p)$ and $\left(M_{n}(p)\right)^{-1}$. All the nonzero elements in $M_{n}(p)$ are ' 1 's whereas inside
$\left(M_{n}(p)\right)^{-1} 3^{n}$ of the nonzero elements are ' 1 's and the rest are ' 2 's.

Property 4. From Definition 9 and the relations between the ternary fastest LI transform matrices of classes Y1 and $\mathrm{Z} 1$ presented in [6], it can be established that

$$
\begin{gathered}
M_{(Y 1.1) n}=M_{n}(0) \\
M_{(Y 1.4) n}=M_{n}\left(6^{n}-1\right) \cdot P_{n}^{6^{n}-1} \\
M_{(Z 1.1) n}=M_{n}\left(6^{n-1}-1\right) \\
M_{(Z 1.4) n}=M_{n}\left(5 \cdot 6^{n-1}\right) \cdot P_{n}^{6^{n}-1} .
\end{gathered}
$$

Property 5. Let $f\left(\overrightarrow{x_{n}}\right)$ be an $n$-variable ternary switching function with the truth vector $\vec{F}$. Then there are $3^{n}-2^{n-1}$ spectral coefficients in the spectrum of $f\left(\overrightarrow{x_{n}}\right)$ based on $M_{n}(p)$ whose values are equal to the values of the truth vector elements, i.e., their values can be directly obtained from $\vec{F}$ without any additions or multiplications. Furthermore, if $\overrightarrow{F_{1}}$ is defined as the subset of the truth vector elements whose values affect the values of the $2^{n}-1$ spectral coefficients that need to be calculated, $\overrightarrow{F_{1}}$ has $2^{n}$ elements.

Property 6. All possible ternary fastest LI matrix with permutation can be divided into $3^{n}$ groups of size $2^{n}$ such that if $S(p)$ is defined as the set of truth vector elements that are directly forwarded to the spectral coefficients of $M_{n}(p)$, then all $M_{n}(p)$ in the same group have identical set $S(p)$. Therefore, the number of elements inside $S(p)$ that have nonzero values gives the minimum number of nonzero elements for the corresponding group of $M_{n}(p)$.

Let the matrix $Z$ be defined as $Z=\left[\begin{array}{lll}0 & 1 & 2 \\ 0 & 2 & 1 \\ 1 & 0 & 2 \\ 2 & 0 & 1 \\ 1 & 2 & 0 \\ 2 & 1 & 0\end{array}\right]$, where the row and column index numbers start from zero. Then any two fastest LI matrices with permutation $M_{n}\left(p_{a}\right)$ and $M_{n}\left(p_{b}\right)$ belong to the same group if

$$
Z_{p_{a_{j}}, 1}=Z_{p_{b_{j}}, 1} \text { for } j=2,3, \ldots, n
$$

and

$$
Z_{p_{a_{j}}, 2}=Z_{p_{b_{j}}, 2} \text { for } j=1 \text {, }
$$

where

$$
<p_{a}>_{6}=<p_{a_{n}}, p_{a_{n-1}}, \ldots, p_{a_{1}}>
$$


$<p_{b}>_{6}=<p_{b_{n}}, p_{b_{n-1}}, \ldots, p_{b_{1}}>$.

\section{Generalized ternary fastest LI transforms}

The ternary fastest LI transforms with permutation defined in Section 3 can be further extended into a wider set of ternary fastest LI transforms by allowing the permutation to be located either in one side of the butterfly diagrams or between the butterfly diagram stages and by allowing the butterfly diagram stages to be reordered such as it has been done for binary fastest LI transforms [9]. The resulting ternary LI transforms are called generalized ternary fastest LI transforms. As reordering and permutation do not incur any additional cost, the computational cost of the generalized ternary fastest LI transforms are the same as the known ternary fastest LI transforms.

Definition 10. Let $M_{n}^{\theta}(\varphi, \sigma, p)$ denote a generalized ternary fastest LI transform of dimension $3^{n} \times 3^{n}$ with ordering $\varphi$, permutation position $\sigma(1 \leq \sigma \leq n+1)$, and permutation number $p \quad\left(0 \leq p \leq 6^{n}-1\right)$. Then $M_{n}^{\theta}(\varphi, \sigma, p)$ is defined as

$M_{n}^{\theta}(\varphi, \sigma, p)= \begin{cases}\left(\prod_{j=n}^{\sigma} K_{n, \varphi_{j}}^{\theta}\right) \cdot P_{n}^{p} \cdot\left(\prod_{j=\sigma-1}^{1} K_{n, \varphi_{j}}^{\theta}\right), & \text { if } \sigma \neq n+1 \\ P_{n}^{p} \cdot \prod_{j=n}^{1} K_{n, \varphi_{j}}^{\theta}, & \text { otherwise, }\end{cases}$

where $\theta \in\{1,2,4,5\}$ and $K_{n, j}^{\theta}$ and $P_{n}^{p}$ have been defined in Property 1 and Definition 8, respectively.

Property 7. The ordering $\varphi$ is an $n$-digit string in which every digit takes values from 1 to $n$ and no two different digits in it are allowed to have the same values,

$$
\varphi=<\varphi_{n}, \varphi_{n-1}, \ldots, \varphi_{1}>,
$$

where $\varphi_{i} \in\{1,2, \ldots, n\}$ and $\varphi_{i}=\varphi_{j}$ iff $i=j(1 \leq i, j \leq n)$.

Property 8. Clearly, the inverse of $M_{n}^{\theta}(\varphi, \sigma, p)$ is simply

$$
M_{n}^{\theta}(\varphi, \sigma, p)= \begin{cases}\left(\prod_{j=1}^{\sigma-1}\left(K_{n, \varphi_{j}}^{\theta}\right)^{-1}\right) \cdot\left(P_{n}^{p}\right)^{-1} \cdot\left(\prod_{j=\sigma}^{n}\left(K_{n, \varphi_{j}}^{\theta}\right)^{-1}\right), & \text { if } \sigma \neq n+1 \\ \left(\prod_{j=1}^{n}\left(K_{n, \varphi_{j}}^{\theta}\right)^{-1}\right) \cdot\left(P_{n}^{p}\right)^{-1}, & \text { otherwise. }\end{cases}
$$

Property 9. Any two generalized ternary fastest LI matrices $M_{n}^{\theta_{1}}\left(\varphi_{1}, \sigma_{1}, p_{1}\right)$ and $M_{n}^{\theta_{2}}\left(\varphi_{2}, \sigma_{2}, p_{2}\right)$ are identical when $\theta_{1}=\theta_{2}, \sigma_{1}=\sigma_{2}, p_{1}=p_{2}$, and $S_{1}=\left\{\varphi_{1 j} \mid 1 \leq j \leq \sigma_{1}-1\right\}=S_{2}=\left\{\varphi_{2 j} \mid 1 \leq j \leq \sigma_{2}-1\right\}$.

Property 10. By (19), (21), and (32), it can be derived that

and

$$
\begin{aligned}
& M_{n}^{4}(\varphi, \sigma, p)=R_{2}{ }^{2}\left(M_{n}^{1}\left(\varphi, \sigma, p^{\prime}\right)\right) \\
& M_{n}^{5}(\varphi, \sigma, p)=R_{2}{ }^{2}\left(M_{n}^{2}\left(\varphi, \sigma, p^{\prime}\right)\right),
\end{aligned}
$$

where $<p>_{6}=<p_{n}, p_{n-1}, \ldots, p_{1}>, p^{\prime}{ }_{j}=0,2,1,4,3$, and 5 if $p_{j}=0,1,2,3,4$, and 5 , respectively, and $<p^{\prime}>_{6}=<p_{n}^{\prime}, p_{n-1}^{\prime}, \ldots, p_{1}^{\prime}>$.

\section{Experimental results}

The calculation of the spectra based on all $M_{n}(p)$ and generalized ternary fastest LI transforms $M_{n}^{\theta}(\varphi, \sigma, p)$ have been implemented in MATLAB and run for several binary benchmark functions that have been modified to represent ternary functions. The translation from binary to ternary cases has been done by changing every two input (output) bits in binary files to an input (output) symbol in ternary files. If the number of input and/or output variables is odd, then a zero bit is first added behind the binary cubes to make it even. For input (output), -- is converted to,- 00 is converted to 0,01 is converted to 1 , 10 is converted to 2 , and 11 is ignored (converted to 0 ). The resulting numbers of nonzero spectral coefficients inside the spectra of each ternary input function based on $M_{n}(0), M_{n}\left(6^{n}-1\right), M_{n}\left(6^{n-1}-1\right)$, and $M_{n}\left(5 \cdot 6^{n-1}\right)$ are listed in Table 1. Recall that those ternary fastest LI transforms with permutation correspond to the known ternary fastest LI transforms. In addition, the number of nonzero spectral coefficients for each input function based on all $M_{n}(p)$ are compared and the minimum number is shown in the rightmost column of Table 1. Based on the numbers in Table 1, it can be seen that for some ternary functions $M_{n}(p)$ reduces the number of terms required to represent them, which leads to faster calculation of the output value, for example for con1, rd84, 9sym, and alu4.

In Table 2, the resulting minimum numbers of nonzero spectral coefficients that can be obtained by each type of $M_{n}^{\theta}(\varphi, \sigma, p)$ are shown. Comparing the numbers in Tables 1 and 2, it can be observed that for some ternary functions $M_{n}^{\theta}(\varphi, \sigma, p)$ can give more compact representations than $M_{n}(p)$ in terms of smaller number of nonzero spectral coefficients. Since $M_{n}(p)$ is a special 
case of $M_{n}^{\theta}(\varphi, \sigma, p)$, the minimum number of spectral coefficients based on all $M_{n}^{\theta}(\varphi, \sigma, p)$ is never larger that that based on $M_{n}(p)$. This can be clearly seen from Table 2 .

\section{Table 1. Number of nonzero spectral coefficients for $M_{n}(p)$}

\begin{tabular}{|l|l|l|l|l|l|}
\hline \multirow{2}{*}{$\begin{array}{c}\text { Input } \\
\text { filename }\end{array}$} & \multicolumn{5}{|c|}{ Number of nonzero spectral coefficients } \\
\cline { 2 - 6 } & $M_{n}(0)$ & $M_{n}\left(6^{n}-1\right)$ & $M_{n}\left(6^{n-1}-1\right)$ & $M_{n}\left(5 \cdot 6^{n-1}\right)$ & $\begin{array}{c}\text { Optimum } \\
M_{n}(p)\end{array}$ \\
\hline xor5 & 10 & 10 & 10 & 10 & 9 \\
\hline con1 & 45 & 46 & 45 & 47 & 42 \\
\hline squar5 & 16 & 17 & 17 & 16 & 16 \\
\hline z5xp1 & 53 & 53 & 53 & 53 & 53 \\
\hline inc & 52 & 51 & 51 & 52 & 51 \\
\hline rd84 & 49 & 50 & 49 & 50 & 43 \\
\hline misex1 & 52 & 53 & 53 & 52 & 51 \\
\hline ex5 & 81 & 81 & 81 & 81 & 77 \\
\hline 9sym & 116 & 123 & 125 & 116 & 107 \\
\hline clip & 153 & 157 & 156 & 153 & 150 \\
\hline apex4 & 162 & 161 & 161 & 162 & 157 \\
\hline ex1010 & 178 & 179 & 179 & 178 & 174 \\
\hline alu4 & 2179 & 2179 & 2179 & 2179 & 2153 \\
\hline misex3 & 2156 & 2161 & 2161 & 2156 & 2150 \\
\hline
\end{tabular}

Table 2. Minimum number of nonzero spectral coefficients for $M_{n}^{\theta}(\varphi, \sigma, p)$

\begin{tabular}{|l|l|l|l|l|}
\hline $\begin{array}{c}\text { Input } \\
\text { filename }\end{array}$ & $M_{n}^{1}(\varphi, \sigma, p)$ & $M_{n}^{2}(\varphi, \sigma, p)$ & $M_{n}^{4}(\varphi, \sigma, p)$ & $M_{n}^{5}(\varphi, \sigma, p)$ \\
\hline xor5 & 8 & 8 & 8 & 8 \\
\hline con1 & 41 & 41 & 38 & 39 \\
\hline squar5 & 15 & 15 & 16 & 15 \\
\hline z5xp1 & 52 & 52 & 51 & 51 \\
\hline inc & 47 & 47 & 45 & 46 \\
\hline rd84 & 42 & 43 & 41 & 43 \\
\hline misex1 & 47 & 46 & 47 & 45 \\
\hline ex5 & 76 & 77 & 75 & 75 \\
\hline 9 sym & 103 & 103 & 107 & 107 \\
\hline clip & 144 & 144 & 145 & 145 \\
\hline apex4 & 155 & 155 & 153 & 153 \\
\hline ex1010 & 174 & 174 & 174 & 174 \\
\hline
\end{tabular}

\section{Conclusion}

Extension of the known ternary fastest LI transforms [6] to generate new classes of ternary fastest LI transforms with the same lowest computational cost have been presented. Several properties and relations for the transforms have also been given. The presented properties and relations can be used to reduce the time and computing resources required to obtain the most compact polynomial representation for a ternary function based on all the ternary fastest LI transforms.

The theory presented in this paper may be of interest not only to researchers working in the area of ternary functions but also in other areas where mathematical models of ternary expansions and functions are used. The corresponding polynomial expansions over $\mathrm{GF}(3)$ can be used not only as bases for bio-orthogonal systems but also as the mathematical apparatus to analyze the stability of finite automata giving more flexibility than the known results for binary dynamic systems [5]. A unified approach to the generation of butterfly structures presented here can also be of interest for researchers developing multiresolution digital signal processing systems using unconventional applications of butterfly decomposition techniques [10].

\section{References}

[1] R. S. Stankovic, M. R. Stojic, and M. S. Stankovic, Recent Developments in Abstract Harmonic Analysis with Applications in Signal Processing, Science Publisher, Belgrade, 1996.

[2] S. Agaian, J. Astola, and K. Egiazarian, Binary Polynomial Transforms and Nonlinear Digital Filters, Marcel Dekker, New York, 1995.

[3] J. Astola and R. S. Stankovic, "Signal Processing Algorithms and Multiple-Valued Logic Design Methods", Proc. 36th IEEE Int. Symp. on Multiple-Valued Logic, Singapore, May 2006, CD publication.

[4] L. P. Yaroslavsky and M. Eden, Fundamentals of Digital Optics, Birkhauser, Boston, 1996.

[5] G. P. Gavrilov and A. A. Sapozhenko, Problems and Exercises on Course of Discrete Mathematics, Science, Moscow, 1992, in Russian.

[6] B. J. Falkowski and C. Fu, "Fastest Classes of Linearly Independent Transforms over GF(3) and Their Properties", IEE Proc. Computers and Digital Techniques, vol. 152, no. 5, pp. 567-576, Sept. 2005.

[7] P. Davio, J. P. Deschamps, and A. Thayse, Discrete and Switching Functions, George and McGraw-Hill, New York, 1978.

[8] R. S. Stankovic and J. T. Astola, Spectral Interpretation of Decision Diagrams, Springer-Verlag, New York, 2003.

[9] S. Rahardja, B. J. Falkowski, and C. C. Lozano, "Fastest Linearly Independent Transforms over GF(2) and Their Properties", IEEE Trans. on Circuits and Systems I, vol. 52, no. 9, pp. 1832-1844, Sept. 2005.

[10] A. Drygajlo, "Butterfly Orthogonal Structure for Fast Transforms, Filter Banks and Wavelets", Proc. 17th IEEE Int. Conf. on Acoustic, Speech, and Signal Processing, San Francisco, USA, March 1992, pp. 81-84. 